ISSN: 2386-3919

ISSN electrónico: 2386-3927

DOI: http://dx.doi.org/10.14201/et2015332123150

\title{
E-LEARNING Y COMUNICACIÓN ORAL Y ESCRITA. UNA PERSPECTIVA SOBRE EL DISEÑO, LA IMPLEMENTACIÓN Y EL IMPACTO ACADÉMICO EN EL CONTEXTO UNIVERSITARIO
}

\author{
E-learning and Oral and Written Communication. \\ A perspective on the design, implementation and \\ academic impact in the university context
}

E-learning et communication orale et écrite.

Un point de vue sur la conception, la mise en ceuvre et l'impact académique dans le contexte universitaire

\author{
Mauro MARINO JIMÉNEZ \\ Universidad San Ignacio de Loyola. Lima. Perú \\ Correo-e:maurosmj@gmail.com; mmarino@usil.edu.pe
}

Recibido: 16-12-2014; Aceptado: 30-04-2015; Publicado: 30-10-2015

BIBLID [2386-3927 (2015) 33, 2; 123-150]

Ref. Bibl. MAURO MARINO JIMÉNEZ. E-learning y Comunicación Oral y Escrita. Una perspectiva sobre el diseño, la implementación y el impacto académico en el contexto universitario. Enseñanza \& Teaching, 33, 2-2015, 123-150.

RESUMEN: El presente estudio analiza la experiencia desarrollada en la asignatura de Comunicación Oral y Escrita en un sistema de educación virtual para adultos en la Universidad San Ignacio de Loyola de Lima (Perú), a través de un estudio cuasiexperimental pretest y postest. Esta propuesta cuenta con una revisión del programa virtual en un entorno andragógico, la evaluación del diseño de acuerdo con la postura de diversos investigadores, las principales características de los productos 
generados por los estudiantes, el impacto académico propiamente dicho y la opinión estudiantil. El resultado de este análisis evidencia que no hay una diferencia significativa en el desarrollo de las competencias lingüísticas en los sistemas presencial y virtual; asimismo, revela la posibilidad de incorporar el desarrollo de competencias digitales en el entorno andragógico antes descrito; finalmente, estos resultados evidencian la posibilidad de un desarrollo promisorio de la educación virtual en el contexto universitario peruano.

Palabras clave: aprendizaje a distancia; competencias lingüísticas; andragogía; competencias digitales.

SUMMARY: This study analyzes the experience developed in the course of Comunicación Oral y Escrita in a virtual system of adult education at the Universidad San Ignacio de Loyola in Lima (Perú) through a quasi-experimental pre-test and posttest study. This proposal is a revision of the virtual program in an andragogic environment, the design evaluation according to the position of various researchers, the main features of the products generated by students, the academic impact and the student's opinion. The result of this analysis shows that there is no significant difference in the development of language skills between physical classrooms and virtual systems; also reveals the possibility of incorporating the development of digital skills in the andragogic environment described above. Finally, these results suggest the possibility of a promising development of virtual education in the Peruvian university context.

Key words: e-learning; linguistics competences; andragogy; digital skills.

RÉSUMÉ: Cette étude analyse l'expérience acquise dans le cadre de Comunication orale et écrite dans un système virtuel de éducation des adultes à l'Université San Ignacio de Loyola à Lima (Pérou) à travers un pré-test et post-test étude quasi-expérimentale. Cette proposition est une révision du programme virtuel dans un environnement andragogique, l'évaluation de la conception en fonction de la position des différents chercheurs, les principales caractéristiques des produits générés par les étudiants, l'impact académique et l'opinion de l'étudiant. Le résultat de cette analyse montre qu'il n'y a pas de différence significative dans le développement des compétences linguistiques entre classes physiques et des systèmes virtuels; révèle aussi la possibilité d'intégrer le développement des compétences digitales dans l'environnement andragogique. Enfin, ces résultats suggèrent la possibilité d'un développement prometteur de l'éducation virtuelle dans le contexte universitaire péruvien.

Mots clés: e-learning; compétences communicatifs; andragogie; compétences numériques.

\section{INTRODUCCIÓN}

El Perú, con 140 universidades en funcionamiento (la mayoría fundadas a partir del año 2000), se erige con un significativo segundo puesto a nivel 
sudamericano en la creación de estas instituciones. A esta medida se puede agregar que el primer puesto de Brasil, con 197, tiene una población casi siete veces mayor (Monzón, 2014). Estas cifras, sin embargo, no han podido ser contrastadas con investigaciones que estimen su éxito académico, o que planteen una evaluación de los nuevos programas implementados en los últimos años, tales como las carreras para adultos y la educación virtual. Más aún, la discreta presencia de solo una universidad peruana entre las cincuenta primeras de Latinoamérica (la Pontificia Universidad Católica del Perú) hace indispensable una mirada honesta sobre los ejemplos de esta oferta educativa (Top Universities, 2014).

Durante los últimos años, el postulado de educación a lo largo de toda la vida se ha manifestado en distintos ámbitos como una necesidad fundamental de las sociedades actuales y en relación con escenarios laborales cambiantes (Delors, 1996). Algunos ejemplos de esto pueden encontrarse en programas universitarios dirigidos a adultos, como se demuestra en España, a través de las pruebas de acceso a grados universitarios para mayores de 25 o 45 años (Ministerio de la Presidencia, 2008), el Programa de Educación para Adultos en Puerto Rico (2015) y las carreras universitarias para personas con experiencia laboral en Perú. Sin embargo, para conocer estas propuestas, resulta conveniente, en primer lugar, comprender sus diferencias con la pedagogía, revisar brevemente la literatura y tener en cuenta algunos ejemplos de implementación.

Por otra parte, los sistemas de educación virtual han proliferado en distintas partes del mundo con el auspicio de universidades con una significativa presencia internacional. Todo ello, sumado a la portabilidad de la información, la conectividad y la navegación geográfica a través de GPS (Global Positioning System), amplía un horizonte de oportunidades que estaba restringido al salón de clases, la biblioteca o la asesoría docente de forma presencial. En tal sentido, tanto la industria editorial como los proveedores de plataformas virtuales o LMS (Learning Management Systems) han llegado a la conclusión de que sus productos deben funcionar con la misma efectividad que en los equipos de escritorio o los libros impresos. Gracias a la Web Semántica se permite el entendimiento de un mismo sistema o programa en distintos dispositivos, pero con interfaces adecuadas para sus dimensiones y capacidad. Casos actuales de esto pueden encontrarse en aplicaciones como Google Drive (https://drive.google.com/), DropBox (https://www.dropbox.com) y Prezi (www.prezi.com) las cuales, además de permitir un trabajo de edición desde múltiples entornos, disponen de la alternativa de trabajar colaborativamente entre diversas cuentas asociadas a un mismo producto y de manera síncrona.

Dentro de la comunidad científica, existe un creciente interés por los beneficios que, potencialmente, puede traer el uso de las Tic y los sistemas de educación virtual. Sin embargo, también se señala que no por el hecho de emplear tecnologías se disfrutará gratuitamente de ellos.

Por tal motivo, diversos estudios relacionados con el tema presentado tienen relación con tres elementos cruciales para lograr una buena implementación: 
- La medición de diversos indicadores (tales como las competencias adquiridas, aceptación del programa y el impulso de habilidades relacionadas con las actividades desarrolladas), para brindar una perspectiva más amplia sobre las necesidades atendidas por el sistema.

- Propuestas de mejora metodológica sobre la base de los resultados obtenidos en aspectos cualitativos y cuantitativos.

- Acciones que logren una mayor participación del estudiante en su propia actividad de aprendizaje.

Algunos ejemplos de estudios recientes denotan las características ya mencionadas.

El estudio de Padilla y Gómez (2014), relacionado con la incorporación de las TIC para la enseñanza de las Ciencias Sociales, se fundamenta en la evaluación de la metodología b-learning a través de la observación de clases, entrevistas aplicadas al docente y encuestas al alumnado. Esta visión cualitativa generó una perspectiva sobre la situación actual en el centro educativo analizado y propuso la gestión de una mayor participación de recursos, capacitación docente y una evaluación posterior de aspectos cuantitativos.

Chibás-Ortiz, Barroto-Carmona y De-Almeida Santos (2014) pusieron énfasis en las diferencias entre un entorno de aprendizaje tradicional y uno creativo, a través de una investigación que observa la necesidad de combinar estratégicamente los distintos recursos y estrategias disponibles, tales como los medios de información (escritos, auditivos, audiovisuales), las actividades on-line y off-line, la comunicación síncrona y asíncrona y el uso orgánico que se le da a todas ellas. Tal grado de implementación determinó mejoras significativas en aspectos como la tasa de retención $(81,33 \%$ contra $57,14 \%)$, el grado de satisfacción $(85,71 \%$ frente a $52,38 \%)$ y la creatividad (76,19\% en lugar de $38,09 \%)$.

Martín y Martínez (2014) desarrollaron, a partir de la necesidad de una comunicación apropiada en el entorno universitario, un sistema de evaluación de escritura publicada electrónicamente en el que docentes y estudiantes tendrían la obligación de calificar y asignar un puntaje. Esta actividad consiguió diversos beneficios: empoderar a estudiantes y docentes para el comentario y evaluación de productos afines a su quehacer académico, comprender la escritura como una herramienta para comunicar el conocimiento adquirido y permitir un desarrollo equilibrado de la misma en toda la población estudiantil.

Gallardo, Marqués y Bullen (2015), a través de un conjunto de entrevistas semiestructuradas a estudiantes universitarios, ponen de manifiesto la necesidad de identificar las herramientas tecnológicas que la población utiliza. Asimismo, relativizan el postulado de considerar que una generación emplea de manera homogénea (en tipo, uso y eficiencia) los mismos recursos tecnológicos, ya que, si bien existen ciertas preferencias a nivel internacional, también es determinante, en un programa educativo mediado por las TIC, el conocimiento de cuáles son las más asociadas a una población determinada. 
E-LEARNING Y COMUNICACIÓN ORAL Y ESCRITA. UNA PERSPECTIVA SOBRE EL DISEÑO,

LA IMPLEMENTACIÓN Y EL IMPACTO ACADÉMICO EN EL CONTEXTO UNIVERSITARIO

Estos breves ejemplos sugieren la visibilidad e importancia que tienen la educación de adultos y las nuevas tecnologías en la educación contemporánea, y serán motivo para que en este artículo se analicen los niveles de desarrollo de competencias lingüísticas en sistemas presenciales y virtuales. Sin embargo, antes de ello, se revisarán brevemente los conceptos presentados.

\section{IMPLEMENTACIÓN DE LA EDUCACIÓN VIRTUAL EN UN ENTORNO ANDRAGÓGICO}

A continuación, se expondrá un recuento sobre las características de la educación para adultos y la idoneidad de la educación virtual en dicho entorno.

\subsection{Educación para adultos}

A diferencia de la educación basada en contenidos -como se dio en la pedagogía durante la mayor parte del siglo Xx-, la educación para adultos ha desarrollado una perspectiva de educación con un fuerte énfasis en la solución de problemas de contexto real, el aprovechamiento de experiencias del educando, el refuerzo de la actividad laboral a través de la educación autorregulada y la capacidad que el adulto tiene para transformar sus habilidades sobre la base de determinadas motivaciones (Instituto de Tecnologías Educativas, 2014; Knowles, 2011; Marcus, 2003).

A propósito de la educación de adultos, cabe citar la siguiente propuesta de la UNESCO (1976):

La expresión educación de adultos designa la totalidad de los procesos organizados de educación, sea cual sea el contenido, el nivel y el método, sean formales o no formales, ya sea que prolonguen o reemplacen la educación inicial dispensada en las escuelas y universidades, y en forma de aprendizaje profesional, gracias a los cuales, las personas consideradas como adultos por la sociedad a la que pertenecen, desarrollan sus aptitudes, enriquecen sus conocimientos, mejoran sus competencias técnicas o profesionales o les da una nueva orientación, y hacen evolucionar sus actitudes o su comportamiento en la doble perspectiva de un enriquecimiento integral del hombre y una participación en un desarrollo socioeconómico y cultural equilibrado e independiente.

Como se señala en la cita, al sumar los elementos interrelacionados en la educación de adultos con el acceso a más oportunidades, se ofrece también una apertura significativa para un aprendizaje informal, en el que los conocimientos de los pares juegan un rol decisivo (Jarche, 2012).

Para que este proceso de enseñanza-aprendizaje sea fructífero, varios autores manifiestan perspectivas complementarias.

Según Dickinson (1992) el aporte que ofrecen las TIC para una educación a lo largo de toda la vida es de vital importancia, ya que contribuyen de forma sustantiva a la interacción entre personas, a mejoras en el estado de países en vías de desarrollo y al aprendizaje fuera del currículo. Al respecto, declara lo siguiente: 
MAURO MARINO JIMÉNEZ

E-LEARNING Y COMUNICACIÓN ORAL Y ESCRITA. UNA PERSPECTIVA SOBRE EL DISEÑO, LA IMPLEMENTACIÓN Y EL IMPACTO ACADÉMICO EN EL CONTEXTO UNIVERSITARIO

As the educational establishment undergoes incremental change, informal learning is escalating through new communications networks. Fifth generation lightware communications are increasing transmission capacity a hundredfold. Rural and third-world development will be accelerated exponentially by electronic networks, two-way communications systems, and the use of expert systems. The whole world will soon be linked by telecomputers that will offer revolutionary new ways of networking and communicating interactively.

Esta postura, anticipada en su época, se ve corroborada con la idoneidad que tienen las TiC en la actualidad, así como en las nuevas alternativas que se plantean para conseguir una educación al alcance de todos.

Billington (1996) plantea la educación de adultos como una forma de incrementar la madurez, ya que ejerce una fuerte influencia en el desarrollo del carácter, el juicio moral, las relaciones interpersonales, el control de los impulsos y el autoconocimiento.

Para que esto tenga efecto, las características del aprendizaje deben ser particularmente distintas de las que se ofrecen en programas pedagógicos, aunque coincidan en algunas de estas.

Entre las medidas sugeridas por el autor, se encuentran las siguientes:

- Generar un ambiente de tranquilidad y soporte para el estudiante.

- Brindar políticas de libertad intelectual que disponga la creatividad y experimentación.

- Ofrecer un trato horizontal de las áreas académicas hacia los adultos.

- Empoderar a los estudiantes para que asuman responsabilidad por su propio aprendizaje.

- Ofrecer situaciones de desafío intelectual.

- Plantear actividades participativas asociadas con situaciones de trabajo.

- Preparar mecanismos de realimentación para conocer la calidad del servicio que ofrece el área académica.

Autonomía, desafío, respeto y aprecio de la participación estudiantil resultan elementos congruentes con una adecuada metodología, y en consonancia con una visión abierta de los conocimientos (Cloutier, 2010; Gauntlett, 2011; Siemens, 2010).

Hermanson (1996) considera que, aunque la educación para adultos se ha masificado en los últimos años, no necesariamente implica que todos los programas ofrecidos sean adecuados. Asimismo, resultados de su investigación demuestran que un aprendizaje social y que atienda el propio desarrollo supera positivamente al conseguido de forma instrumental, enfocado solamente en la adquisición de información. En tal sentido, los propios participantes declaran que los espacios de interacción y de autodescubrimiento son los que resultan más significativos.

Esta postura de Hermanson sugiere que es absolutamente indispensable incorporar los aspectos emotivos, en los que una atmósfera positiva potencia la labor intelectual. Esto, además de convertirse en una experiencia más internalizada, 
E-LEARNING Y COMUNICACIÓN ORAL Y ESCRITA. UNA PERSPECTIVA SOBRE EL DISEÑO,

LA IMPLEMENTACIÓN Y EL IMPACTO ACADÉMICO EN EL CONTEXTO UNIVERSITARIO

produce transformaciones que conjugan buenos resultados académicos y la satisfacción de los alumnos.

El estudio de Markus (2003) participa de la discusión sobre la capacidad del adulto para aprender y disminuir la pérdida de las funciones cognitivas a través de hábitos saludables y estudios adicionales a su formación adquirida en las primeras etapas de su vida.

En su investigación, dicho autor demuestra que la experiencia y el aprendizaje pueden hacer que el cerebro cambie su funcionamiento. Esto, no solo en relación con la adquisición de saberes, sino que, con un programa adecuado, los participantes manifiestan mejoras en aspectos relacionados con el aprecio de múltiples perspectivas y su capacidad de análisis.

Como se evidencia en los comentarios de los distintos investigadores, la educación de adultos se desenvuelve en múltiples líneas de acción, en consonancia con beneficios para quienes participan de ella (mejora económica, transformación del funcionamiento del cerebro y participación ciudadana), en la optimización del programa propiamente dicho (gracias a la horizontalidad y apertura de la institución educativa en la que participa) y el desarrollo de la sociedad en la que se desenvuelve. En tal sentido, la edad, actividades y responsabilidades del adulto son significativas cuando reciben la influencia positiva de una educación formulada para gestionar sus habilidades con mayor precisión y aprovechamiento.

En el contexto peruano, se ha generado una creciente oferta de estos programas, con modelos educativos adaptados a la realidad y necesidades de los participantes. Tal es el caso de la Universidad San Ignacio de Loyola (USIL), la Universidad Esan (UE), la Universidad Peruana de Ciencias Aplicadas (UPC), la Pontificia Universidad Católica del Perú (PUCP) y la Universidad Femenina del Sagrado Corazón (UNIFE).

Como se declaró previamente, este artículo relatará la experiencia en el primer ejemplo mencionado.

La UsiL, con sede principal en la ciudad de Lima, ha implementado un conjunto de programas denominado Carreras para Personas con Experiencia Profesional (CPEL), el cual se caracteriza por un público con las siguientes características:

a) Edad mínima de 24 años.

b) Experiencia laboral mínima de 4 años.

c) En caso de convalidación, debe haber cursado un mínimo de 36 créditos en estudios universitarios.

En este contexto, los estudiantes del programa CPEL evidencian una relación directa con las necesidades y características quienes ya han tenido experiencia y estabilidad en el ámbito laboral, y se desenvuelven en búsqueda de un nivel de reconocimiento sobre su actuación en la sociedad (Instituto de Tecnologías Educativas, 2014; Maslow, 1943).

El desarrollo de CPEL se gestó en tres etapas: 
a) Creación, a inicios del año 2008, a través de los grados presenciales de Administración de Empresas, Marketing y Gestión Comercial, Negocios Internacionales e Ingeniería Empresarial y de Sistemas.

b) Ampliación, a partir del 2011, de sistemas semipresencial y virtual para las asignaturas de estudios generales y el grado completo de Administración de Empresas.

c) Integración, a partir del 2014, de los tres sistemas en las cuatro carreras antes mencionadas, además de Contabilidad Empresarial e Ingeniería Industrial.

De acuerdo con lo anterior, los ocho años de existencia del programa han ampliado una oferta educativa en consonancia con las necesidades del público al que atiende. Ello se refleja en un crecimiento del número de ingresantes por periodo académico de 113 a más de 800, así como en una ampliación de la oferta educativa fuera de la ciudad de Lima, para atender a ciudades como Arequipa, Chiclayo, Cusco, Huancayo, Piura y Trujillo (Figura 1).

Al ser CPEL un programa descentralizado por medio de la virtualización de sus asignaturas, es conveniente conocer qué tipos de aportes puede representar en su desarrollo formal.

\section{FIGURA 1}

Alcance de la propuesta educativa de CPEL al 2014

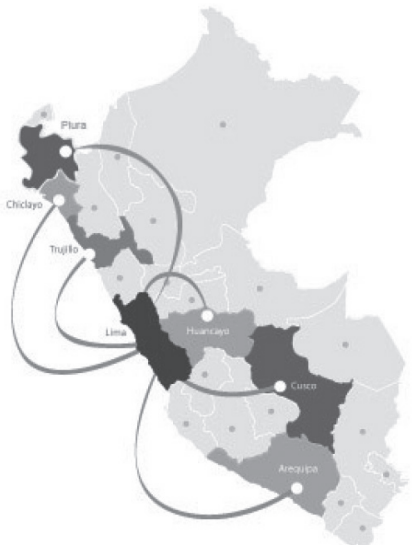

Fuente: http://www.usil.edu.pe/cpelvirtual/programa.htm\#propuesta.

\subsection{Uso de la tecnología en la educación}

De acuerdo con diversos autores, las tecnologías de la información y de la comunicación (TIC) han dejado de ser solamente un medio para pasar a convertirse 
E-LEARNING Y COMUNICACIÓN ORAL Y ESCRITA. UNA PERSPECTIVA SOBRE EL DISEÑO,

LA IMPLEMENTACIÓN Y EL IMPACTO ACADÉMICO EN EL CONTEXTO UNIVERSITARIO

en un agente de cambio para manifestar la identidad, necesidades y manifestaciones del ser humano y la transformación de la sociedad que este forme (Cloutier, 2010; Gauntlett, 2011; Vázquez-Cano y Sevillano, 2011; Vázquez-Cano, 2012). En tal sentido, un sistema e-learning no significa solamente una plantilla o esquema sobre el cual se deba inscribir un determinado conjunto de actividades, sino también que este, a través de distintos elementos dinámicos, disponga el aporte y la reflexión del estudiante adulto, con la finalidad de facilitar su aprendizaje. En tal sentido, los viejos roles de autor, editor y sistema educativo como elementos estables han sufrido cuestionamientos en los últimos años, para plantear nuevas didácticas, más relacionadas con las necesidades cambiantes y en los que los conocimientos no se encuentran solamente en las personas (Belderrain, 2013; Cloutier, 2010; Siemens, 2010, Vázquez-Cano, 2012).

Una de estas teorías es el conectivismo planteado por Siemens, quien lo define como

la integración de principios explorados por las teorías del caos, redes, complejidad y autoorganización. El aprendizaje es un proceso que ocurre en el interior de ambientes difusos de elementos centrales cambiantes -que no están por completo bajo control del individuo-. El aprendizaje (definido como conocimiento aplicable) puede residir fuera de nosotros (en el interior de una organización o una base de datos), está enfocado a conectar conjuntos de información especializada, y las conexiones que nos permiten aprender más tienen mayor importancia que nuestro estado actual de conocimiento (2010: 84).

Esta postura sugiere una forma de trabajo compatible con lo antes descrito, pues parte de la idea de la incapacidad del ser humano para retener individualmente los conocimientos y los incluye en un proceso de cambio permanente, sobre el cual cada agente se caracteriza por su participación en redes antes que por la acumulación de conocimientos.

A continuación, se ofrecen otros aportes a la propuesta educativa en un sistema virtual.

Para Casamayor (2008: 95), resulta importante la capacidad de alternar las actividades individuales con el trabajo colaborativo, considerando a este último indispensable tanto para el desarrollo disciplinar como a capacidades para comunicarse, asumir tareas, negociar y organizar equipos. Esta postura es particularmente significativa cuando se trata de garantizar la permanencia de los estudiantes en la asignatura elegida, ya que los hace partícipes de una determinada comunidad.

Para Aparici (2010: 10), la pluralidad de estilos de aprendizaje y habilidades informáticas hace necesario el paso previo de identificar a la población para plantear la viabilidad de un sistema educativo determinado. Esto significa que parte del éxito de un determinado diseño implica un análisis previo de las características intelectuales y culturales de los participantes.

Cloutier (2010), Gabelas (2010) y Osuna (2010) sugieren consolidar las funciones para cada agente (docente o estudiante) dentro de la práctica educativa. Este 
último, asimismo, recomienda el uso de hipertextos, hipermedia y multimedia para acercar a los distintos tipos de participantes y sus estilos de aprendizaje.

Según Vázquez-Cano y Sevillano (2011) existe una marcada importancia en una educación basada en productos por parte del cuerpo estudiantil. Estrategias para conseguir este propósito son los talleres creativos, gestión de proyectos, guías de navegación y diarios de clase.

Para López y Villafañe (2011) el rol de la tecnología no solo sirve como una herramienta, sino también para la redefinición de la sociedad a través de indicadores como el comportamiento social y el pensamiento. Por tal motivo, la educación se sumerge y transforma en dicho proceso.

Para Vázquez-Cano (2012, 2013) las Tic adquieren un papel determinante en la complejidad del centro educativo, dado que su correcto uso permite una mayor horizontalidad para atender objetivos comunes y dinamiza el proceso de mejora colectiva. En tal sentido, las tecnologías aparecen tanto en la didáctica como en la gestión del centro educativo. Asimismo, aparece también en la necesidad de determinar su efectividad a través de instrumentos previamente validados.

Hernández (2013) y Shepherd (2013) destacan la importancia de llevar la información a distintas latitudes y circunstancias gracias al aporte de los dispositivos móviles. Ello también pone en evidencia la capacidad de estos procesadores para considerar la viabilidad de una determinada plataforma.

Esta rápida revisión de distintos autores sobre el aporte de las Tic sugiere una participación de estas en diversos aspectos: el desarrollo de un sistema e-learning, el análisis de la situación actual para el desarrollo de proyectos educativos eficaces, la optimización de la gestión educativa, el impacto social, el planteamiento de modelos adecuados con las necesidades específicas, etc. Finalmente, hay una alta congruencia entre la capacidad de conexión actual y la propuesta de basar el aprendizaje en productos elaborados por los estudiantes.

A continuación, se describirán algunos aspectos relacionados con el desarrollo específico del programa.

\subsection{Implementación}

La propuesta de trabajo se centró en la asignatura de Comunicación Oral y Escrita. Un curso que plantea el desarrollo de competencias lingüísticas, tales como comprensión de la información, expresión oral y expresión escrita. En tal sentido, se manifiesta lo que el estudiante conseguirá al culminar la propuesta:

Comprende y maneja los diferentes niveles del lenguaje a través de la producción de diversos textos escritos, orales y audiovisuales acordes con su especialidad, elaborados con coherencia y propiedad, y utilizando correctamente la normativa del castellano.

La hipótesis del presente estudio es que los efectos de la modalidad virtual son similares a la versión presencial. En tal sentido, estos serán observados en los 
siguientes aspectos: (a) la coherencia del planteamiento teórico, (b) el impacto académico en el desarrollo de competencias lingüísticas y (c) la aceptación de la modalidad virtual por parte de los estudiantes.

Para explicar la coherencia del planteamiento teórico, se explicará la concepción del diseño y el desarrollo de actividades y su vinculación con lo sugerido por literatura relacionada con las TIC.

En el trabajo con la asignatura de Comunicación Oral y Escrita, se consideraron unidades interrelacionadas que conducen al desarrollo de tareas coherentes y progresivas, las cuales permiten una internalización gradual de las herramientas tecnológicas disponibles (Casamayor, 2008; Vázquez y Sevillano, 2011). Para ello, se elaboró un plan de siete semanas, con 10 horas de trabajo en cada una de ellas, que conduzca a la comprensión de la escritura, la distinción entre lo denotativo y lo connotativo, la semiótica y el abordaje a la elaboración de exposiciones a través de medios audiovisuales (Figura 2).

FIGURA 2

Red de aprendizaje de la asignatura de Comunicación Oral y Escrita

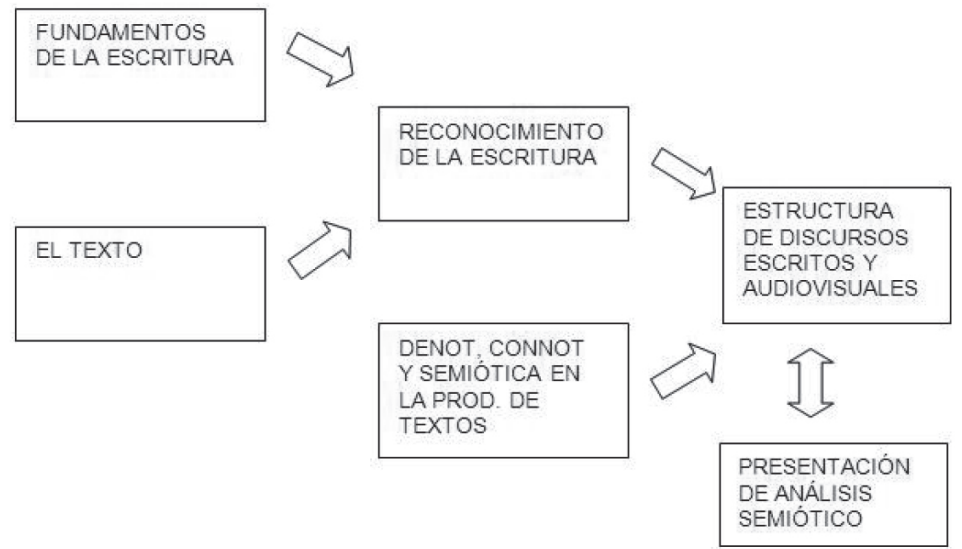

Fuente: Sílabo de Comunicación Oral y Escrita.

El desarrollo de los contenidos en ambas modalidades se llevó a cabo en la plataforma Chamilo versión 1.8.8.6 (www.chamilo.org), la cual ofrece opciones de seguimiento a actividades como foros, tareas, wikis, chat, y desarrollo de contenidos, denominados "Itinerario de aprendizaje». En ellos se puede establecer una relación jerárquica entre contenidos, tales como módulos, unidades o semanas. En este caso, se recurrió a la última opción ya que, al ser asignaturas modulares, resulta más sencillo entender actividades dentro de periodos cronológicos de tiempo. Cada una de las distintas semanas fue cargada previamente a la plataforma; 
sin embargo, se establecieron particularidades en cuanto a los elementos del sistema virtual, ya que, además de adaptar lecciones específicamente para un trabajo predominantemente autónomo, se recurrió a diversas incorporaciones, las cuales se describirán a continuación.

La primera incorporación la constituye una batería de herramientas gratuitas, tales como Prezi (www.prezi.com), Slideshare (www.slideshare.net) y Proprofs (www.proprofs.com), las cuales fueron elegidas para promover un estudio menos lineal y facilitar la adquisición informal de saberes (Figura 3).

FiguRA 3

Ejemplo de Itinerario de aprendizaje de la semana 4

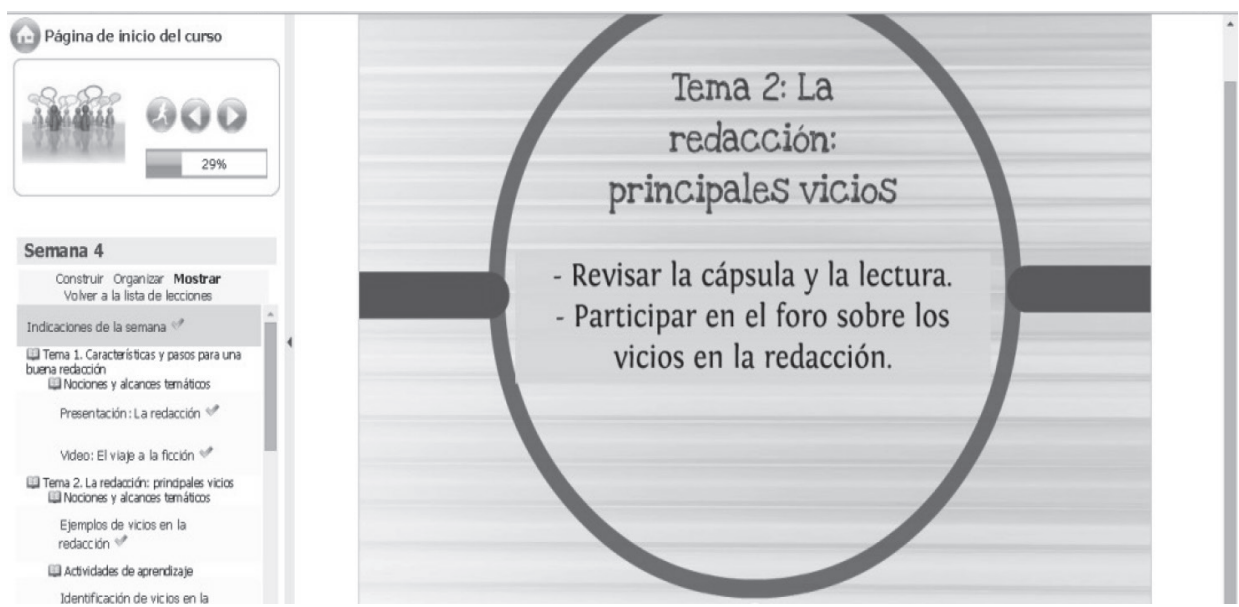

Fuente: Diseño de Comunicación Oral y Escrita en la Plataforma Chamilo.

Por otra parte, la estructura interna de cada semana desarrolla una secuencia que va desde las indicaciones generales por temas, el planteamiento de contenidos (en formato escrito, sonoro o audiovisual) y el desarrollo de actividades de aprendizaje para consolidar y comprobar los saberes (a través de diversas herramientas, tales como foros, wikis y tareas). Asimismo, al final de cada semana se puede examinar el desarrollo de todo lo planteado mediante distintos productos (Tabla 1). 
MAURO MARINO JIMÉNEZ

E-LEARNING Y COMUNICACIÓN ORAL Y ESCRITA. UNA PERSPECTIVA SOBRE EL DISEÑO,

LA IMPLEMENTACIÓN Y EL IMPACTO ACADÉMICO EN EL CONTEXTO UNIVERSITARIO

TABLA 1

Esquema semanal

\begin{tabular}{|c|c|c|c|}
\hline \multicolumn{3}{|c|}{ CATEGORÍA } & SOPORTE \\
\hline \multirow{4}{*}{ INDICACIÓN SEMANAL } & Tema 1 & Contenidos & Textos, audios y videos \\
\cline { 3 - 4 } & Tema 2 & Actividad de aprendizaje & Foros, Wikis o tareas \\
\cline { 2 - 4 } & & Contenidos & Textos, audios y videos \\
\cline { 2 - 4 } & Tema 3 & Actividad de aprendizaje & Foros, Wikis o tareas \\
\cline { 2 - 4 } & & Actividad de aprendizaje & Foros, Wikis o tareas \\
\cline { 2 - 4 } & \multicolumn{2}{|c|}{ Actividad de evaluación } \\
\hline
\end{tabular}

Fuente: Elaboración propia.

Los canales de comunicación propuestos fueron los foros; sin embargo, a pedido de los estudiantes, se dispuso que contaran con el uso de Wikis para potenciar la coordinación de sus actividades grupales. Los Wikis, asimismo, ofrecen otra ventaja: la revisión del historial. De esta forma, pueden observarse las ediciones anteriores de un mismo trabajo, por lo que se hace posible detectar y corregir intrusiones de parte de personas que no tienen relación con un determinado espacio.

Un ejemplo de trabajo ya sistematizado dentro del programa fue el análisis semiótico de un comercial de televisión, en el que se evidencia la convergencia entre lo escrito y lo audiovisual (Figura 4).

FIGURA 4

Ejemplo de trabajo realizado en Wiki

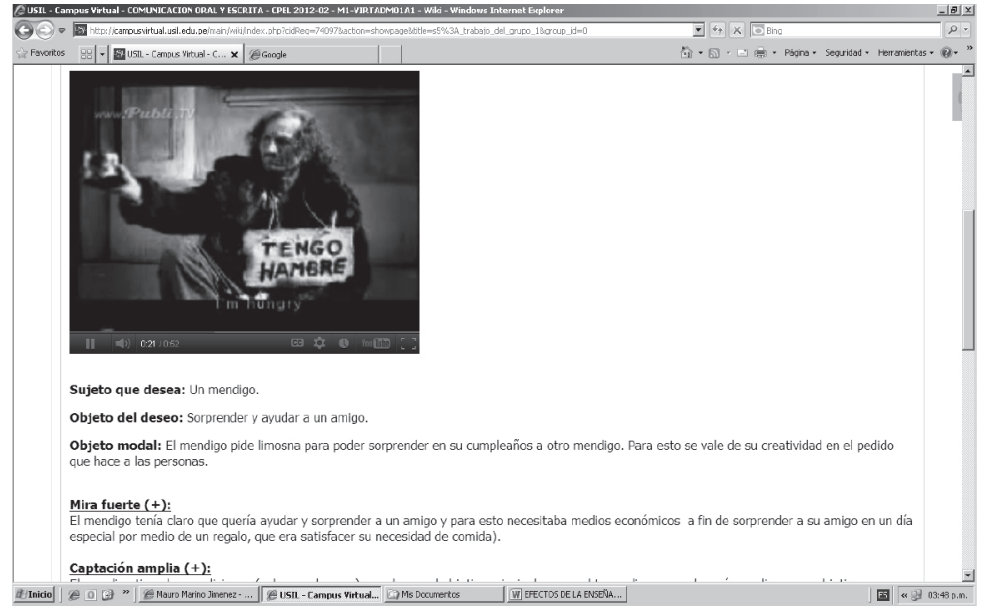

Fuente: Plataforma virtual de Comunicación Oral y Escrita. 
Respecto de los tipos de actividades, se estableció una alternancia entre calificadas y no calificadas. De esta manera, se pudo establecer una mayor libertad para la actuación entre pares, y una motivación a actuar en todas ellas sin temor a ser sancionados por cometer equivocaciones. Esto incluyó el uso de foros, wikis, tareas y autoevaluaciones.

Finalmente, para el desarrollo de auditorías, se implementó un procedimiento de recordatorios, análisis del tiempo de permanencia y actividades realizadas para determinar un mayor acercamiento a aquellos estudiantes que no ingresaron en la plataforma por una determinada cantidad de tiempo.

Por lo observado anteriormente, el programa ofrecido no solo ofrece una rutina de actividades, sino también de interacción, rutas alternativas de aprendizaje y diversidad de medios, tanto en la búsqueda de calificaciones como en la participación legítima, que solamente se busca el beneficio común. Esta primera evidencia ofrece un grado de consonancia con la bibliografía mencionada para referirse a la enseñanza por medio de las TIC y la andragogía.

\subsection{Diferencia de productos}

Además de las pruebas asignadas para el desarrollo de las competencias lingüísticas, a las que se hace referencia en este estudio (Comprensión de la Información y Expresión Escrita), una diferencia significativa entre el grupo de control (presencial) y el grupo experimental (virtual) fue la necesidad de suplir la exposición oral que se presenta al final del curso por una presentación que caracterice la Expresión Oral, y sobre la cual pueda gestionarse un registro calificable.

En tal sentido, el sistema e-learning implementado desarrolló un producto que caracterice la Expresión Oral, pero a través de medios audiovisuales. Esta propuesta, al igual que en el sistema presencial, consistió en un análisis semiótico, pero que pueda desarrollarse a través de registros formales o informales, según la necesidad del trabajo.

Esta actividad permitió reunir el trabajo de distintos grupos de estudiantes (de una misma o distinta ubicación geográfica), quienes no solo pusieron en evidencia su trabajo en expresión oral, sino que también establecen un vínculo con las competencias digitales (Aparici, 2010; Prensky, 2010; Sevillano, 2009). Algunos de estos trabajos se presentan en la Tabla 2. 
E-LEARNING Y COMUNICACIÓN ORAL Y ESCRITA. UNA PERSPECTIVA SOBRE EL DISEÑO, LA IMPLEMENTACIÓN Y EL IMPACTO ACADÉMICO EN EL CONTEXTO UNIVERSITARIO

TABLA 2

Ejemplos de trabajos de Comunicación Oral y Escrita en vídeo

\begin{tabular}{|c|c|}
\hline UBICACIÓN GEOGRÁFICA & DIRECCIÓN ELECTRÓNICA \\
\hline Distintas sedes & http://goo.gl/g2dHrc \\
\hline Misma sede & http://goo.gl/Td8Xvp \\
\hline Diferentes sedes & $\mathrm{http}: / /$ goo.gl/XlCi8s \\
\hline Misma sede & $\mathrm{http}: /$ goo.gl/nbxcl8 \\
\hline
\end{tabular}

Fuente: Elaboración propia.

\section{Metodología}

La hipótesis de este estudio es que los efectos de esta modalidad son similares a los de la versión presencial. Para tal efecto, el trabajo desarrollado se declara predominantemente cualitativo, con diseño cuasiexperimental pretest y postest. Ello, debido a las siguientes razones: (a) las evaluaciones atienden al análisis del desarrollo de las competencias, según los estados inicial y final del programa; (b) aunque se aplicó el análisis a toda la población aludida, esta resulta demasiado restringida en relación con estudios cuantitativos, y (c) los grupos conformados por sus propias preferencias respecto de lo presencial y lo virtual. Este estudio, sin embargo, permite analizar con mayor profundidad otros aspectos, como las ya referidas, y las opiniones de los estudiantes sobre el sistema virtual a través de una encuesta.

\subsection{Características de la muestra}

Los participantes del programa fueron estudiantes de ambos sexos, con edades de 24 años a más, divididos en grupos de 17 y 15 respectivamente, en una muestra no probabilística por conveniencia, tomando en cuenta a todos aquellos que iniciaron y culminaron la asignatura. En cuanto a sus especialidades, se ofrecen variaciones en el grupo de control, con un 64,7\% abocado a Ingeniería de Sistemas, un 23,5\% en Administración de Empresas y un 11,7\% en Negocios Internacionales. En cuanto al grupo experimental, se halló unicidad en Administración de Empresas, debido a la condición de exclusividad que en ese entonces había (ver Tabla 3 y Tabla 4). 
MAURO MARINO JIMÉNEZ

E-LEARNING Y COMUNICACIÓN ORAL Y ESCRITA. UNA PERSPECTIVA SOBRE EL DISEÑO, LA IMPLEMENTACIÓN Y EL IMPACTO ACADÉMICO EN EL CONTEXTO UNIVERSITARIO

TABLA 3

Características del grupo de control

\begin{tabular}{|c|c|c|c|}
\hline & CARRERA & EDAD & SEXO \\
\hline 1 & Ingeniería Empresarial de Sistemas & 39 & Masculino \\
\hline 2 & Administración de Empresas & 25 & Femenino \\
\hline 3 & Administración de Empresas & 29 & Femenino \\
\hline 4 & Ingeniería Empresarial de Sistemas & 54 & Masculino \\
\hline 5 & Ingeniería Empresarial de Sistemas & 40 & Masculino \\
\hline 6 & Ingeniería Empresarial de Sistemas & 24 & Masculino \\
\hline 7 & Ingeniería Empresarial de Sistemas & 47 & Masculino \\
\hline 8 & Ingeniería Empresarial de Sistemas & 35 & Masculino \\
\hline 9 & Negocios Internacionales & 25 & Masculino \\
\hline 10 & Ingeniería Empresarial de Sistemas & 41 & Masculino \\
\hline 11 & Negocios Internacionales & 32 & Masculino \\
\hline 12 & Ingeniería Empresarial de Sistemas & 41 & Masculino \\
\hline 13 & Ingeniería Empresarial de Sistemas & 29 & Masculino \\
\hline 14 & Administración de Empresas & 27 & Masculino \\
\hline 15 & Ingeniería Empresarial de Sistemas & 29 & Masculino \\
\hline 16 & Ingeniería Empresarial de Sistemas & 33 & Masculino \\
\hline 17 & Administración de Empresas & 27 & Masculino \\
\hline & EDAD MEDIA & & 34 \\
\hline
\end{tabular}

Nota: El grupo de control, conformado por 17 participantes, tiene edades que fluctúan entre los 24 y 54 años. Asimismo, se puede señalar coincidencias con el grupo experimental, ya que ofrecen intervalos de edades medias y medianas similares. Finalmente, es destacable la presencia de participantes masculinos (15 de los 17), quienes conforman un 88,2\% del total. Fuente: Elaboración propia. 
MAURO MARINO JIMÉNEZ

E-LEARNING Y COMUNICACIÓN ORAL Y ESCRITA. UNA PERSPECTIVA SOBRE EL DISEÑO,

LA IMPLEMENTACIÓN Y EL IMPACTO ACADÉMICO EN EL CONTEXTO UNIVERSITARIO

TABLA 4

Características del grupo experimental

\begin{tabular}{|c|c|c|c|}
\hline & CARRERA & EDAD & SEXO \\
\hline 1 & Administración de Empresas & 38 & Femenino \\
\hline 2 & Administración de Empresas & 50 & Masculino \\
\hline 3 & Administración de Empresas & 30 & Masculino \\
\hline 4 & Administración de Empresas & 33 & Masculino \\
\hline 5 & Administración de Empresas & 39 & Masculino \\
\hline 6 & Administración de Empresas & 23 & Masculino \\
\hline 7 & Administración de Empresas & 31 & Femenino \\
\hline 8 & Administración de Empresas & 34 & Masculino \\
\hline 9 & Administración de Empresas & 32 & Masculino \\
\hline 10 & Administración de Empresas & 25 & Femenino \\
\hline 11 & Administración de Empresas & 34 & Femenino \\
\hline 12 & Administración de Empresas & 40 & Masculino \\
\hline 13 & Administración de Empresas & 31 & Femenino \\
\hline 14 & Administración de Empresas & & Masculino \\
\hline 15 & Administración de Empresas & & 34 \\
\hline & & Edad media &
\end{tabular}

Nota: El grupo experimental, conformado por 15 participantes, cuenta con edades que fluctúan entre los 25 y 50 años. Asimismo, presenta intervalos similares a los del grupo de control y corroborados con la semejanza entre sus medias y medianas. Finalmente, es destacable la presencia de participantes masculinos (11 de los 15), quienes conforman un 73,3\% bastante cercano a la proporción de sus pares. Fuente: Elaboración propia.

\subsection{Instrumentos de evaluación y procedimientos de análisis}

El estudio fue planteado a través de pretest y postest, tomando en cuenta el desarrollo de las competencias lingüísticas de los grupos de control y experimental. A ello se agregó un mecanismo secundario de medición, consistente en una encuesta sobre la aceptación que tiene la asignatura en sistema e-learning. En este apartado se describirá las características y validación de ambos instrumentos.

Las pruebas constaron de 10 preguntas cada una, en relación a las dimensiones de Expresión Escrita y Comprensión de la Información.

A continuación, se ofrecerán la estructura de las pruebas de entrada y de salida (Tabla 5), así como la encuesta aplicada (Tabla 6). 
TABLA 5

Estructura de las pruebas de entrada y de salida

\begin{tabular}{|c|c|c|c|c|}
\hline DIMENSIONES & INDICADORES & ÍTEMS & PUNTOS & NIVEL \\
\hline \multirow{2}{*}{$\begin{array}{l}\text { Expresión escrita: El } \\
\text { estudiante analiza la } \\
\text { normativa, coherencia } \\
\text { y adecuación } \\
\text { necesarias para una } \\
\text { redacción formal. }\end{array}$} & $\begin{array}{c}\text { 1. Analiza la } \\
\text { normativa en } \\
\text { casos puntuales. }\end{array}$ & $1,2,3$ & 6 puntos & \multirow{2}{*}{$\begin{array}{c}\text { Ejemplar: } 11 \text { a } \\
12 \text { puntos } \\
\text { Competente: } 8 \\
\text { a } 10 \text { puntos } \\
\text { En desarrollo: } 3 \\
\text { a } 7 \text { puntos } \\
\text { Inicial: } 0 \text { a } 2 \text { puntos }\end{array}$} \\
\hline & $\begin{array}{l}\text { 2. Analiza la } \\
\text { adecuación y } \\
\text { la coherencia. }\end{array}$ & $4,5,6$ & 6 puntos & \\
\hline \multirow{2}{*}{$\begin{array}{l}\text { Comprensión de } \\
\text { la información: El } \\
\text { estudiante identifica la } \\
\text { intención del autor, así } \\
\text { como las principales } \\
\text { características de } \\
\text { un tipo de texto. }\end{array}$} & $\begin{array}{l}\text { 3. Identifica las } \\
\text { características } \\
\text { del texto. } \\
\end{array}$ & 7,8 & 2 puntos & \multirow{2}{*}{$\begin{array}{l}\text { Ejemplar: } 8 \text { puntos } \\
\text { Competente: } 6 \\
\text { a } 7 \text { puntos } \\
\text { En desarrollo: } 2 \\
\text { a } 5 \text { puntos } \\
\text { Inicial: } 0 \text { a } 1 \text { puntos }\end{array}$} \\
\hline & $\begin{array}{l}\text { 4. Identifica } \\
\text { la intención } \\
\text { del autor. }\end{array}$ & 9,10 & 3 puntos & \\
\hline
\end{tabular}

Nota: Para revisar las pruebas completas, revisar su publicación en http://goo.gl/hhtk1A (anexo 4). Fuente: Elaboración propia.

TABLA 6

Encuesta aplicada

\begin{tabular}{|c|c|}
\hline DIMENSIONES & ÍTEMS \\
\hline Conceptual & 1 y 2 \\
\hline Procedimental & $3,4,5,6,7$ \\
\hline Actitudinal & 8,9 Y 10 \\
\hline
\end{tabular}

Fuente: Elaboración propia.

Las pruebas fueron validadas a través del coeficiente $\mathrm{V}$ de Aiken, el cual analiza la relevancia de cada ítem respecto de un dominio en $N$ jueces. En tal sentido, para conseguir la aprobación, el coeficiente debe estar entre 0,80 y 1,00, siendo 1,00 la expresión de un acuerdo total entre los expertos. En ambos casos el resultado fue igual o mayor a lo necesario (Tablas 7 y 8). 
MAURO MARINO JIMÉNEZ

E-LEARNING Y COMUNICACIÓN ORAL Y ESCRITA. UNA PERSPECTIVA SOBRE EL DISEÑO,

LA IMPLEMENTACIÓN Y EL IMPACTO ACADÉMICO EN EL CONTEXTO UNIVERSITARIO

TABLA 7

Intervalos de confianza y $\mathrm{V}$ de Aiken para la prueba de entrada

\begin{tabular}{|c|c|c|c|c|}
\hline \multirow{2}{*}{ DIMENSIONES } & \multirow{2}{*}{ ÍTEMS } & \multirow{2}{*}{ V DE AIKEN } & \multicolumn{2}{|c|}{ INTERVALOS DE CONFIANZA } \\
\cline { 4 - 5 } & & & Límite inferior & Límite superior \\
\hline \multirow{4}{*}{ EXPRESIÓN ESCRITA } & ÍTEM 1 & 0,93 & 0,7 & 0,99 \\
\cline { 2 - 5 } & ÍTEM 2 & 0,93 & 0,7 & 0,99 \\
\cline { 2 - 5 } & ÍTEM 3 & 0,93 & 0,7 & 0,99 \\
\cline { 2 - 5 } & ÍTEM 5 & 0,80 & 0,55 & 0,93 \\
\cline { 2 - 5 } & ÍTEM 4 & 0,93 & 0,7 & 0,99 \\
\cline { 2 - 5 } & ÍTEM 6 & 1,00 & 0,8 & 1,00 \\
\hline \multirow{4}{*}{$\begin{array}{c}\text { COMPRENSIÓN DE } \\
\text { LA INFORMACIÓN }\end{array}$} & ÍTEM 7 & 0,87 & 0,63 & 0,96 \\
\cline { 2 - 5 } & ÍTEM 8 & 0,87 & 0,63 & 0,96 \\
\cline { 2 - 5 } & ÍTEM 9 & 0,93 & 0,7 & 0,99 \\
\cline { 2 - 5 } & ÍTEM 10 & 0,93 & 0,7 & \\
\hline
\end{tabular}

Fuente: Elaboración propia.

TABLA 8

Intervalos de confianza y $\mathrm{V}$ de Aiken para la prueba de salida

\begin{tabular}{|c|c|c|c|c|}
\hline \multirow{2}{*}{ DIMENSIONES } & \multirow{2}{*}{ ÍTEMS } & \multirow{2}{*}{ V DE AIKEN } & \multicolumn{2}{|c|}{ INTERVALOS DE CONFIANZA } \\
\cline { 4 - 5 } & & & Límite inferior & Límite superior \\
\hline \multirow{4}{*}{ EXPRESIÓN ESCRITA } & ÍTEM 1 & 1,00 & 0,8 & 1,00 \\
\cline { 2 - 5 } & ÍTEM 2 & 0,93 & 0,7 & 0,99 \\
\cline { 2 - 5 } & ÍTEM 3 & 0,87 & 0,63 & 0,96 \\
\cline { 2 - 5 } & ÍTEM 5 & 0,93 & 0,7 & 0,99 \\
\cline { 2 - 5 } & ÍTEM 4 & 0,93 & 0,7 & 0,99 \\
\cline { 2 - 5 } & ÍTEM 6 & 0,93 & 0,7 & 0,99 \\
\hline \multirow{4}{*}{$\begin{array}{c}\text { COMPRENSIÓN DE } \\
\text { LA INFORMACIÓN }\end{array}$} & ÍTEM 7 & 0,80 & 0,55 & 0,93 \\
\cline { 2 - 5 } & ÍTEM 8 & 0,80 & 0,55 & 0,93 \\
\cline { 2 - 5 } & ÍTEM 9 & 1,00 & 0,8 & 1,00 \\
\cline { 2 - 5 } & ÍTEM 10 & 0,87 & 0,63 & 0,96 \\
\hline
\end{tabular}

Fuente: Elaboración propia.

En complemento al trabajo realizado por estas evaluaciones, se elaboró una encuesta no comparativa para descubrir el desarrollo de dimensiones relacionadas 
con el aprendizaje de la asignatura (dimensión conceptual), aspectos metodológicos (dimensión procedimental) y la actitud que genera el programa (dimensión actitudinal). En este caso, se mostrará la encuesta completa (Tabla 9) y los resultados de validación (Tabla 10).

TABLA 9

Encuesta dirigida a estudiantes

\section{CURSO} FECHA

1. Luego de la experiencia en Comunicación oral y escrita en modalidad virtual, considera que su aprendizaje del curso fue:

\begin{tabular}{|c|c|c|c|c|}
\hline Excelente & Muy bueno & Regular & Malo & Muy malo \\
\hline
\end{tabular}

2. En comparación con los cursos presenciales, el aprendizaje en modalidad virtual fue:

\begin{tabular}{|l|l|l|l|l} 
Mucho mejor & Mejor & Igual & Peor & Mucho peor \\
\hline
\end{tabular}

3. Aprender a manejar la plataforma virtual resultó:

\begin{tabular}{|c|c|c|c|c|}
\hline Muy fácil & Fácil & $\begin{array}{c}\text { De mediana } \\
\text { dificultad }\end{array}$ & Difícil & Muy difícil \\
\hline
\end{tabular}

4. La comunicación por medio de la plataforma y el correo electrónico funcionó de manera:

\begin{tabular}{|c|c|c|c|c|}
\hline Excelente & Muy buena & Buena & Regular & Deficiente \\
\hline
\end{tabular}

5. La disposición de presentaciones, videos y enlaces como herramientas para el aprendizaje resultaron:

\begin{tabular}{|c|c|c|c|c|}
\hline Imprescindibles & $\begin{array}{c}\text { De mucha } \\
\text { utilidad }\end{array}$ & Útiles & Poco útiles & Prescindibles \\
\hline
\end{tabular}

6. El trabajo con las autoevaluaciones como herramienta para el aprendizaje del curso fue:

\begin{tabular}{|c|c|c|c|c|}
\hline Imprescindible & $\begin{array}{c}\text { De mucha } \\
\text { utilidad }\end{array}$ & Útil & Poco útil & Prescindible \\
\hline
\end{tabular}

7. El trabajo con los foros, tareas y wikis como herramientas para el aprendizaje del curso resultó:

\begin{tabular}{|c|c|c|c|c|}
\hline Imprescindible & $\begin{array}{c}\text { De mucha } \\
\text { utilidad }\end{array}$ & Útil & Poco útil & Prescindible \\
\hline 8. & En general, podría decir que la modalidad virtual resultó: & \\
\hline Muy agradable & Agradable & Aceptable & Desagradable & $\begin{array}{c}\text { Muy } \\
\text { desagradable }\end{array}$ \\
\hline
\end{tabular}

9. Si le ofrecieran llevar otros estudios en modalidad virtual su respuesta sería:

\begin{tabular}{|c|c|c|c|c|}
\hline Muy agradable & Agradable & Tal vez & $\begin{array}{c}\text { Posiblemente } \\
\text { no }\end{array}$ & $\begin{array}{c}\text { Definitivamente } \\
\text { no }\end{array}$ \\
\hline
\end{tabular}

10. Escriba alguna sugerencia para mejorar el trabajo con esta modalidad:

Fuente: Elaboración propia. 
MAURO MARINO JIMÉNEZ

E-LEARNING Y COMUNICACIÓN ORAL Y ESCRITA. UNA PERSPECTIVA SOBRE EL DISEÑO,

LA IMPLEMENTACIÓN Y EL IMPACTO ACADÉMICO EN EL CONTEXTO UNIVERSITARIO

TABLA 10

Intervalos de confianza y $\mathrm{V}$ de Aiken para la encuesta de opinión

\begin{tabular}{|c|c|c|c|c|}
\hline \multirow{2}{*}{ DIMENSIONES } & \multirow{2}{*}{ ÍTEMS } & \multirow{2}{*}{ V DE AIKEN } & \multicolumn{2}{|c|}{ INTERVALOS DE CONFIANZA } \\
\cline { 4 - 5 } & & & Límite inferior & Límite superior \\
\hline \multirow{3}{*}{ CONCEPTUAL } & ÍTEM 1 & 0,93 & 0,7 & 0,99 \\
\cline { 2 - 5 } & ÍTEM 2 & 0,93 & 0,7 & 0,99 \\
\hline \multirow{5}{*}{ PROCEDIMENTAL } & ÍTEM 3 & 1 & 0,8 & 1 \\
\cline { 2 - 5 } & ÍTEM 4 & 1 & 0,7 & 1 \\
\cline { 2 - 5 } & ÍTEM 5 & 1 & 0,8 & 1 \\
\cline { 2 - 5 } & ÍTEM 6 & 0,93 & 0,7 & 0,99 \\
\cline { 2 - 5 } & ÍTEM 7 & 1 & 0,8 & 1 \\
\hline \multirow{3}{*}{ ACTITUDINAL } & ÍTEM 8 & 0,87 & 0,63 & 0,96 \\
\cline { 2 - 5 } & ÍTEM 9 & 0,93 & 0,7 & 0,99 \\
\cline { 2 - 5 } & ÍTEM 10 & 1 & 0,7 & \\
\hline
\end{tabular}

Fuente: Elaboración propia.

Las evaluaciones de las competencias de Comprensión de la Información y Expresión Escrita se aplicaron durante la primera y última semana de clases, en adición a las actividades planteadas para cada uno de los sistemas y bajo las mismas restricciones de tiempo.

La encuesta, por otra parte, se formuló de forma no comparativa a través de Google docs, con el propósito de recoger las opiniones sobre el trabajo desempeñado en las aulas virtuales y preservar el anonimato necesario para procurar una opinión no comprometida con el sistema empleado.

\section{Resultados}

A continuación, se presentarán los resultados sobre la base de las competencias analizadas y la opinión estudiantil.

\subsection{Competencias}

La evolución de las competencias de Expresión Escrita y Comprensión de la Información tuvo, en su conjunto, un desarrollo similar. Mientras que el grupo de control pasó de 10,9 a 15,6 en escala vigesimal, el grupo experimental pasó de 11,6 a 15,2. Ello se traduce en porcentajes de desarrollo del 23,1\% y 18\%, respectivamente. En tal sentido, ambos grupos evidenciaron una notable evolución, con una ligera ventaja para el primero, aunque no significativa en términos estadísticos.

A nivel de competencias individuales, se puede precisar un progreso más cercano en la Comprensión de la Información, ya que, con 8 puntos posibles, 
E-LEARNING Y COMUNICACIÓN ORAL Y ESCRITA. UNA PERSPECTIVA SOBRE EL DISEÑO, LA IMPLEMENTACIÓN Y EL IMPACTO ACADÉMICO EN EL CONTEXTO UNIVERSITARIO

se alcanzó un progreso de 4,8 a 7,1 (28,8\%) para el grupo de control y de 4,8 a $6,7(23,8 \%)$ para el grupo experimental (Figura 5). Mientras que en la Expresión Escrita, con 12 puntos totales, la diferencia fue ligeramente mayor: de 6,1 a 8,6 $(20,8 \%)$ para el primero y de 6,8 a $8,5(14,1 \%)$ para el segundo (Figura 6).

Figura 5

Resultados de ambos grupos para la competencia de Comprensión de la información

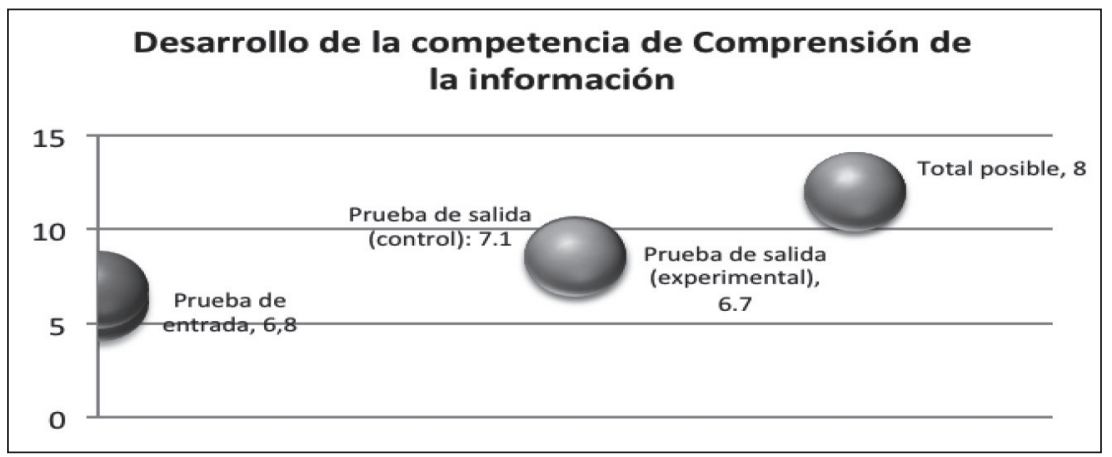

Fuente: Elaboración propia.

FIGURA 6

Resultados de ambos grupos para la competencia de Expresión escrita

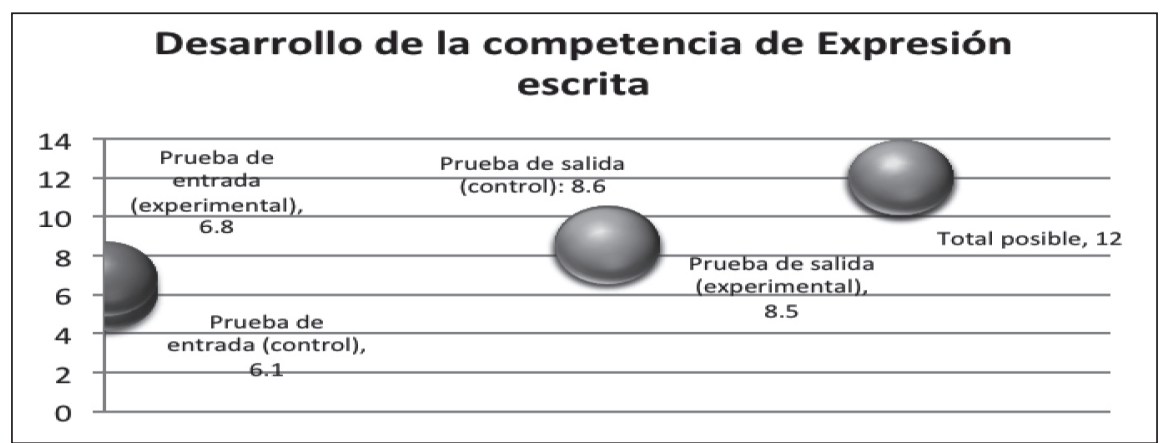

Fuente: Elaboración propia. 
E-LEARNING Y COMUNICACIÓN ORAL Y ESCRITA. UNA PERSPECTIVA SOBRE EL DISEÑO,

LA IMPLEMENTACIÓN Y EL IMPACTO ACADÉMICO EN EL CONTEXTO UNIVERSITARIO

Este resultado refleja lo siguiente:

a) Hay una evolución significativa en ambos grupos, luego de haber pasado por el desarrollo de la asignatura en uno y otro sistema.

b) La competencia de Comprensión de la Información alcanzó un mayor desarrollo en ambos casos, con un $28,8 \%$ y un $23,3 \%$ para el grupo de control y experimental, respectivamente.

c) En cuanto a la competencia de Expresión Escrita, su progreso fue menor: un $20,8 \%$ para el grupo de control y un $14,1 \%$ para el grupo experimental. Esta diferencia en el progreso sugiere una mejora en el planteamiento de actividades relacionadas directamente con dicha competencia.

Aunque los resultados de esta investigación se basan en el desarrollo de las competencias lingüísticas, resulta de utilidad saber no solamente si el programa resulta eficiente, sino también si este genera aceptación por parte de los estudiantes. Por tal motivo, se presenta también una visión sobre este aspecto, a través de una encuesta distribuida de forma anónima.

Los resultados de dicha encuesta fueron los siguientes:

a) Sobre la calidad de aprendizaje, 1 opinó que fue excelente, 10 señalaron que fue muy bueno y 4 , que resultó regular. En tal sentido, los dos primeros grupos suman un $73,3 \%$ del total de encuestados.

b) Respecto de una pregunta abocada a comparar su aprendizaje con la modalidad presencial, 4 participantes señalaron que fue mucho mejor; 9, que fue mejor, y 2 que resultó igual. En tal sentido, un 86,7\% encuentra el sistema virtual como favorable.

c) La tercera pregunta, orientada a la dificultad para el manejo de la plataforma, obtuvo como resultado que 3 participantes la consideraron muy fácil; 6 la percibieron como fácil, y 5, de mediana dificultad. Es decir, un 60\% no encontraron mayor dificultad en el aprendizaje de las herramientas.

d) Respecto de la comunicación, 3 la percibieron como excelente, 6 muy buena, 4 buena y 2 regular. Esta pregunta fue la primera que registró un $13,3 \%$ en un sentido parcialmente negativo.

e) Respecto de herramientas que transmiten contenidos, tales como presentaciones, vídeos y enlaces, 2 las percibieron como imprescindibles, 10 como de mucha utilidad y 3 señalaron que fueron útiles. Es decir, un $80 \%$ estuvieron satisfechos.

f) En cuanto a las autoevaluaciones como herramientas para el aprendizaje, 5 participantes las vieron como imprescindibles, 5 de mucha utilidad, para 4 fueron útiles y solo 1 consideró que resultaron poco útiles. En tal sentido, las percepciones en un nivel sobresaliente fueron de un $60 \%$, en un nivel neutral de un $26,7 \%$ y en un nivel negativo un $6,7 \%$.

g) Respecto del uso de herramientas para distinto tipo de actividades, 2 estudiantes las encontraron imprescindibles, 8 de mucha utilidad, 4 las vieron 
E-LEARNING Y COMUNICACIÓN ORAL Y ESCRITA. UNA PERSPECTIVA SOBRE EL DISEÑO, LA IMPLEMENTACIÓN Y EL IMPACTO ACADÉMICO EN EL CONTEXTO UNIVERSITARIO

útiles y 1 las consideró poco útiles. En tal sentido, lo muy positivo está en un $66,7 \%$, lo neutral en un $26,7 \%$ y lo negativo en un $6,7 \%$.

h) Sobre su opinión general respecto del sistema e-learning, 3 participantes lo consideraron muy agradable, para 6 fue agradable y 6 lo percibieron aceptable. En tal sentido, para un $60 \%$ el trabajo estuvo en lo positivo.

i) Sobre la posibilidad de llevar nuevamente asignaturas virtuales, 10 definitivamente lo harían, 3 posiblemente sí y 2 tal vez optarían por dicho sistema. En este caso, lo positivo se encuentra en un 86,7\%, frente a un 13,3\% que se halla en lo neutral.

j) Asimismo, en la única pregunta abierta, en relación con sugerencias de mejora, se planteó lo siguiente:

- La plataforma debería funcionar en distintos navegadores.

- Se debería incorporar otra herramienta para proyectar anuncios y recordatorios sobre las actividades.

- Asimismo, se deberían introducir espacios de comunicación sincrónica, tales como videoconferencia.

A partir de lo expuesto, se puede afirmar que existe una evidente mayoría de estudiantes que manifiesta su aceptación sobre el trabajo desarrollado en el sistema e-learning de la asignatura de Comunicación Oral y Escrita (ver Figura 7). Sin embargo, también resulta esclarecedor que existen ciertos aspectos relacionados con la dimensión procedimental (tales como la comunicación, las autoevaluaciones y el uso de herramientas). Esto resulta importante para el fortalecimiento del trabajo en el sistema virtual.

\section{FIGURA 7}

Valoración de los estudiantes según las dimensiones conceptuales, procedimentales y actitudinales

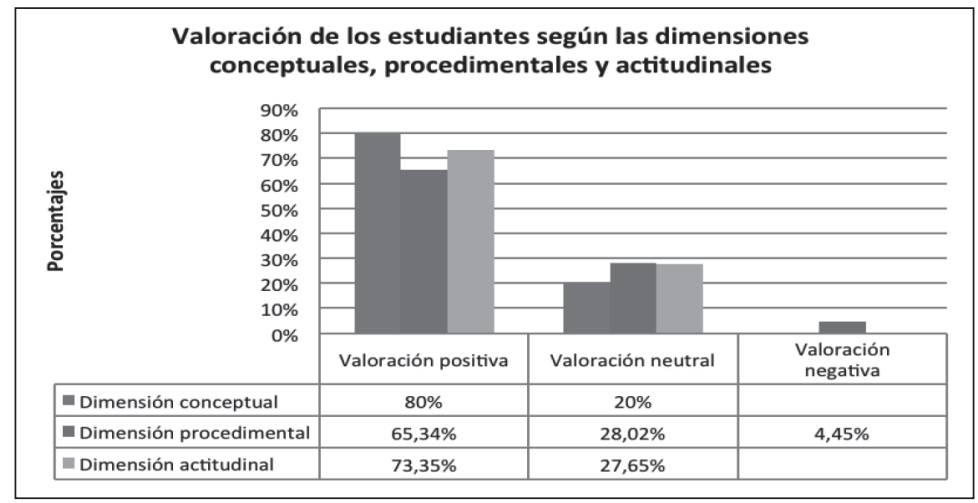

Fuente: Elaboración propia. 


\section{CONCLUSIONES Y SUGERENCIAS}

En el presente estudio se ha analizado la coherencia teórica, el desarrollo de competencias lingüísticas y la opinión estudiantil sobre la base de una propuesta de enseñanza virtual para adultos, a través de un diseño cuasiexperimental pretest y postest, a lo que se acompañó una valoración de la opinión estudiantil. En tal sentido, se han ofrecido resultados promisorios, sobre los cuales se puede mencionar lo siguiente:

En primer lugar, el diseño observado refiere una coherencia interna, distanciada de rutas lineales de aprendizaje. Por otro lado, coincide con la propuesta de enseñanza para adultos y la educación virtual, pues sugiere una participación conjunta en el manejo de conocimientos, un aprovechamiento de la experiencia estudiantil, actividades autorreguladas y productos afines a distintos medios de comunicación.

El desarrollo de las competencias lingüísticas no ha evidenciado una diferencia significativa entre ambos grupos, aunque se puede observar la necesidad de establecer un mayor énfasis en la competencia de Expresión Escrita para ambos, ya que el progreso en esta dimensión resultó menor.

La opinión del grupo experimental evidencia una orientación hacia la valoración positiva, la cual llega a un $80 \%$ en la dimensión conceptual y a $73 \%$ en lo actitudinal. Sin embargo, en lo que respecta a la procedimental, se recomienda un fortalecimiento del sistema de comunicación, junto con la posibilidad de establecer actividades síncronas.

Sobre la base de los resultados obtenidos, caben las siguientes sugerencias:

Si bien es cierto que el diseño del programa resulta adecuado para los propósitos planteados, existen limitaciones para que pueda practicarse una evaluación del progreso de las competencias planteadas, como es el caso de la expresión oral. Por lo tanto, convendría reformular la propuesta en actividades interrelacionadas para darle sostenibilidad e indicadores que orienten sus mejoras.

En las competencias y opinión evaluadas se sugiere que el programa puede ser sostenible en el tiempo. Por lo tanto, cabe la posibilidad de que este tipo de análisis pueda practicarse en el resto de disciplinas que constituyen los grados virtuales. Esto, también, acompañado de una población más numerosa, la cual permita estudios cuantitativos.

\section{LIMITACIONES Y PROSPECTIVA}

A raíz del presente estudio, y en consonancia con una necesidad de mejorar la propuesta educativa en un entorno andragógico para potenciar la competencia de Expresión Escrita y el grado de satisfacción sobre los procedimientos de la asignatura de Comunicación Oral y Escrita, se ha incorporado la realización de una serie de cambios. 
En primer lugar, se ha incorporado el desarrollo de proyectos de redacción, a ser trabajados en ambos sistemas y a lo largo de la asignatura. La presentación de estos proyectos se da en tres oportunidades durante el periodo académico.

La presentación de este proyecto de redacción incluye la sustentación oral de los mismos durante las distintas entregas de la asignatura, ya sea de forma presencial o virtual, según la modalidad empleada. Ello, con el propósito de conocer el desarrollo de la competencia de Expresión Oral a través de una forma sistemática, y no solo al final del curso.

Finalmente, se ha incorporado el empleo de videoconferencias semanales para el sistema e-learning, con el propósito de fortalecer los sistemas de comunicación y darle valor a la propuesta de actividades síncronas.

Gracias a la implementación de estas medidas, se espera lograr un fortalecimiento tanto en el desarrollo de competencias lingüísticas como en la propuesta educativa. Todo ello, con el propósito de servir de referente para otros sistemas e-learning a nivel universitario en el Perú y afianzar la relación entre los adultos y el empleo adecuado de herramientas digitales.

\section{REFERENCIAS BIBLIOGRÁFICAS}

Aparici, R. (Comp.) (2010). Conectados en el ciberespacio. Madrid: Universidad Nacional de Educación a Distancia.

Belderrain, J. (2013). Impacto de las TIC en la enseñanza de lenguas extranjeras, en su comunidad educativa e industria: estado de la cuestión. Disponible en http://www.canal. uned.es/mmobj/index/id/16524.

Casamayor G. (2008). La formación on-line. Una mirada integral sobre el e-learning, b-learning, Barcelona: Graó.

Chibás-Ortiz, F.; Borroto-Carmona, G. y De-Almeida-Santos, F. (2014). Gestión de la creatividad en entornos virtuales de aprendizaje colaborativos: Un proyecto corporativo de EAD. Revista Científica de Educomunicación, 43, XXII, 143-151.

Cloutier, J. (2010). Historia de la comunicación. En R. Aparici. Conectados en el ciberespacio (pp. 45-50). Madrid: Universidad Nacional de Educación a Distancia.

Congreso de la República (2014). Artículo 47. Educación a distancia. En Ley Universitaria. Disponible en http://cde.3.elcomercio.pe/doc/0/0/9/1/1/911198.pdf?ref-nota_ politica\&ft-contenido.

CPEL (2014). CPEL Carreras para Personas con Experiencia Profesional. Disponible en http:// www.usil.edu.pe/cpel.

Delors, J. (1996). La educación encierra un tesoro. Disponible en http://www.rau.edu.uy/ docs/delors_s.pdf.

Gabelas, J. (2010). Educación en la red. Algunas falacias, promesas y simulacros. En R. Aparici (Comp.). Conectados en el ciberespacio. Madrid: Universidad Nacional de Educación a Distancia.

Gallardo, E.; Marqués, L. y Bullen, M. (2015). El estudiante en la educación superior: Usos académicos y sociales de la tecnología digital. RUSC. Universities and Knowledge Society Journal, 12 (1), 25-37. doi: http://dx.doi.org/10.7238/rusc.v12i1.2078 
E-LEARNING Y COMUNICACIÓN ORAL Y ESCRITA. UNA PERSPECTIVA SOBRE EL DISEÑO,

LA IMPLEMENTACIÓN Y EL IMPACTO ACADÉMICO EN EL CONTEXTO UNIVERSITARIO

Gauntlett, D. (2011). Making is connecting. Cambridge: Polity Press.

Hernández, G. (2013). Aprendizaje con smartphones: Tendencias móviles en un mundo cambiante. Disponible en https://canal.uned.es/mmobj/index/id/16536.

Instituto de Tecnologías Educativas (2014). Módulo 3: Fundamentos Pedagógicos. En Fundamentos de la Educación de Personas Adultas. Disponible en http://www.ite.educacion.es/formacion/materiales/125/cd/modulos_pdf/fundamentos_m3_b.pdf.

IPE (2012). No más competencia en la educación universitaria de mala calidad. Disponible en http://www.ipe.org.pe/comentario-diario/14-12-2012/no-mas-competencia-en-laeducacion-universitaria-de-mala-calidad.

Jarche, H. (2012). Informal Learning, the 95\% Solution. Disponible en http://www.jarche. com/2012/01/informal-learning-the-95-solution/.

Knowles, M. W.; Holton, E. F. III y Swanson, R. A. (2011). The adult learner (7 ${ }^{\text {th }}$ ed.). Houston, TX: Gulf.

López, I. y Villafañe, C. (2011). La integración de las TIC al currículo: propuesta práctica. Razón y Palabra, 74. Disponible en http://www.razonypalabra.org.mx/N/N74/ VARIA74/54LopezV74.pdf.

Maslow, A. (1943; 2000). A Theory of Human Motivation. En Classics in the History of Psychology. Disponible en http://psychclassics.yorku.ca/Maslow/motivation.htm.

Martín, B. y Martínez, M. (2014). La enseñanza de la escritura académica: una intervención a través del currículum. @tic. Revista d'innovació educativa, 13, 12-22.

Ministerio de la Presidencia (2008). Real Decreto 1892/2008, de 14 de noviembre, por el que se regulan las condiciones para el acceso a las enseñanzas universitarias oficiales de grado y los procedimientos de admisión a las universidades públicas españolas. Disponible en https://www.boe.es/buscar/act.php?id-BOE-A-2008-18947.

Monzón, R. (2014). Perú es el segundo país en Sudamérica con más universidades. Perú 21. Viernes 03 de enero de 2014. Disponible en http://peru21.pe/politica/peru-segundopais-sudamerica-mas-universidades-2164015.

Osuna, S. (2010). Interactuantes e interactuados en la Web 2.0. En R. Aparici. Conectados en el ciberespacio (pp. 135-149). Madrid: Universidad Nacional de Educación a Distancia.

Padilla, F. y Gómez, M. (2014). Incorporación de las tecnologías de información y comunicación para la enseñanza de las ciencias sociales. 3C TIC. 11 (3), 197-211.

Prensky, M. (2010). Homo sapiens digital: de los inmigrantes nativos y digitales a la sabiduría digital. En R. Aparici. Conectados en el ciberespacio (pp. 93-106). Madrid: Universidad Nacional de Educación a Distancia.

Programa de Educación para Adultos (2015). Disponible en http://www.de.gobierno.pr/ ofrecimiento-academico/191-educacion-de-adultos/431-programa-de-educacion-paraadultos.

Sevillano, M. L. (Coord.) (2009). Competencias para el uso de las herramientas virtuales en la vida, trabajo y formación permanente. Madrid: Pearson.

Shepherd, R. (2013). La edición de material escolar - una industria en revolución. Disponible en https://canal.uned.es/mmobj/index/id/16532.

Siemens, G. (2010). Conectivismo: una teoría de aprendizaje para la era digital. En R. Aparici. Conectados en el ciberespacio (pp. 77-90). Madrid: Universidad Nacional de Educación a Distancia.

Top Universities (2014). QS Latin American University Rankings 2014. Disponible en http://www.topuniversities.com/university-rankings/latin-american-universityrankings/2014\#sorting-rank+region-+country-+faculty-+stars-false+search-. 
unESCO (1976). Definición. De Actas de la Conferencia General. 19a reunión. Disponible en http://unesdoc.unesco.org/images/0011/001140/114038s.pdf.

Vázquez-Cano, E. (2012). Caos, Complejidad y tecnologías en el centro educativo. Madrid. Saarbrücken: Editorial Académica Española.

Vázquez-Cano, E. (2013). Cómo diseñar una metodología de investigación en didáctica aplicada a las lenguas. Disponible en https://canal.uned.es/mmobj/index/id/16537.

Vázquez-Cano, E. y Sevillano, M. ${ }^{a}$ L. (2011). Educadores en Red. Elaboración de materiales audiovisuales para la enseñanza. Madrid: Ediciones Académicas. 